ENGINEERING NOTES are short manuscripts describing new developments or important results of a.preliminary nature. These Notes cannot exceed 6 manuscript pages and 3 figures a page of text may be substituted for a figure and vice versa. After informal review by the editors, they may be published within a few months of the date of receipt. Style requirements are the same as for regular contributions (see inside back cover).

\section{Periodic Control and the Optimality of Aircraft Cruise}

\author{
Elmer G. Gilbert* and Michael G. Parsons $\dagger$ \\ University of Michigan, Ann Arbor, Mich.
}

\section{Introduction}

$\mathbf{I}_{\mathrm{N}}^{\mathrm{N}}$ $\mathrm{N}$ a recent series of papers ${ }^{1-6}$ the form of minimum-fuel, fixed-range trajectories has been discussed. Depending on the model of aircraft dynamics and the method of analysis, it is possible to come to different conclusions. If the energystate approximation ${ }^{1}$ is used to represent aircraft dynamics, the optimization problem may not have a solution. ${ }^{2,3}$ In this case the infimum of fuel consumption is obtained by a trajectory which consists of three segments: a powered climb, a relaxed-control cruise at constant specific energy (which can be approximated by a rapid cycling of thrust, altitude, and velocity at essentially constant specific energy), and a maximum-range glide. ${ }^{2}$ If a more elaborate model of aircraft dynamics is used (point mass, equilibrium of vertical forces, small flight-path angle), the maximum principle indicates that the cruise segment is a classical steady-state cruise. ${ }^{3}$ However, the classical cruise is a singular arc which does not satisfy the generalized Legendre-Clebsch condition and, thus, cannot be minimizing. ${ }^{4}$ With an even more elaborate model (point mass, small angle of attack) the classical cruise segment satisfies the generalized Legendre-Clebsch condition. ${ }^{5}$ Speyer ${ }^{6}$ shows this analysis to be insufficient in that the steady-state cruise does not satisfy the Jacobi condition. Thus, the existence of an optimal, steady-state cruise is still subject to question, at least for certain types of aircraft and aircraft models.

Similar questions about the optimality of steady-state solutions appeared in the chemical engineering literature of the early 1960s. This led to the examination of time-dependent periodic controls and the development of an extensive theory of optimal periodic control. 7,8 Gilbert ${ }^{9}$ has applied this theory to a simple model of vehicle cruise and found that time-dependent control may increase specific range. This note shows that the periodic control formulation extends to aircraft cruise problems. Thus, the theory of optimal periodic control can be applied to the analysis of the cruise segment. For instance, some of Speyer's results ${ }^{6}$ can be obtained from the $\pi$ criterion. ${ }^{10}$

In what follows the main emphasis is on the energy-state model. It is shown that the relaxed cruise mentioned by Zagalsky et al. ${ }^{2}$ is a relaxed steady-state (RSS) optimum of the type described in the literature of periodic control. $7,8,11$ Two examples are considered: the F-4 aircraft and an idealized model of an aircraft. When the maximum altitude is suitably constrained, it is seen in both examples that oscillatory aircraft motion is likely to reduce fuel consumption in cruise.

Received March 8, 1976; revision received May 17, 1976.

Index Categories: Aircraft Performance; Aircraft Handling, Stability, and Control; Navigation, Control, and Guidance Theory.

*Professor, Department of Aerospace Engineering. During the period in which this research was carried out the author was a Visiting Professor in the Department of Electrical Engineering, The Johns Hopkins University.

HAssistant Professor, Department of Naval Architecture and Marine Engineering.

\section{Formulation of the Optimal Periodic Control Problem}

The model of aircraft motion used here is the same considered in previous papers. ${ }^{3,5,6}$ However, it is convenient to measure the specific energy in terms of equivalent altitude, $E=V^{2} / 2 g+h$, and use range $x$ as the independent variable. This gives the equations:

$$
\begin{aligned}
& \mathrm{d} E / \mathrm{d} x=(W \cos \gamma)^{-1}(T-D(h, V, \alpha)) ; E(0)=E\left(x_{l}\right) \\
& \mathrm{d} \gamma / \mathrm{d} x=\left(V^{2} \cos \gamma\right)^{-1}(L(h, V, \alpha)-W \cos \gamma) ; \gamma(0)=\gamma\left(x_{l}\right) \\
& \mathrm{d} h / \mathrm{d} x=\tan \gamma ; h(0)=h\left(x_{l}\right)
\end{aligned}
$$

where $V$ is speed, $\gamma$ is the flight-path angle, $h$ is the altitude, $T$ is the thrust without afterburner, $\alpha$ is the angle of attack, $D$ is the drag, $L$ is the lift, and $W$ is the weight. The equality of initial and terminal values of $E, \gamma$, and $h$ requires the aircraft motion to be periodic with period $x_{l}$. It is desired to minimize the fuel consumed per unit of $x$. Thus, the cost to be minimized is,

$$
J=\frac{I}{x_{1}} \int_{0}^{x_{I}} \sigma(h, V, T) T(V \cos \gamma)^{-1} \mathrm{~d} x
$$

where $\sigma$ is the thrust specific fuel consumption. In general, there will be additional constraints of the form,

$$
0 \leq h, 0 \leq T \leq T_{\max }(h, V), \alpha \leq \alpha_{\max }(h, V)
$$

The minimization of $J$ with respect to $\alpha, T$, and $x_{I}$ is a standard optimal periodic control (OPC) problem. ${ }^{7,8}$ When $E, \gamma$, $h, \alpha$, and $T$ are assumed to be constant an optimal steadystate (OSS) problem is obtained: Eq. (1) is satisfied with derivatives zero, Eq. (3) holds, $x$, disappears, and $J=J_{S S}=\sigma$ $(h, V, T) T V^{-1}$. This solution of the OSS problem is the classical optimum cruise. A variety of techniques exist for determining if the OPC problem is proper, i.e., OPC has a lower cost than OSS. ${ }^{7-11}$ Here only the relaxed steady-state approach is considered. Because of the form of the velocity set (see Schultz and Zagalsky, ${ }^{3}$ App. A, Sec. 3) this approach proves to be ineffective for the model previously given in Eq. (1).

\section{Energy-State Approximation and Relaxed Steady-State Analysis}

Relaxed steady-state analysis does lead to interesting results when applied to the energy-state model. The periodic control problem corresponding to the energy-state approximation can be obtained from Eqs. (1-3) by assuming $\gamma(x)=0$. This yields

$$
\begin{aligned}
\mathrm{d} E / \mathrm{d} x & =\tilde{T}-\bar{D}(h, E), E(0)=E\left(x_{l}\right) \\
J & =\frac{l}{x_{1}} \int_{0}^{x_{I}} \tilde{f(h, E, \bar{T}) \mathrm{d} x}
\end{aligned}
$$

where

$$
\begin{aligned}
V & =(2 g(E-h))^{1 / 2} ; \bar{T}=W^{-1} T ; \bar{D}(h, E) \\
& =W^{-1} D(h, V, \alpha(h, V)) ; \alpha(h, V)
\end{aligned}
$$

is obtained from

$$
L(h, V, \alpha)=W \text {, and } \overline{f(h, E, \bar{T})}=W \sigma(h, V, W \bar{T}) \bar{T} V^{-l}
$$


The controls $h$ and $\bar{T}$ are constrained by

$$
0 \leq h, 0 \leq \bar{T} \leq \bar{T}_{\max }(h, E), \bar{\alpha}(h, E) \leq 0
$$

where $\bar{T}_{\max }(h, E)=W^{-l} T_{\max }(h, V)$ and, $\bar{\alpha}(h, E) \leq 0$ corresponds to $\alpha(h, V) \alpha_{\max }(h, V)$.

The velocity set for the OPC problem [Eqs. (4-6)] is

$$
\begin{aligned}
\gamma(E) & =\left\{\left(z_{l}, z_{2}\right) ; z_{1}=\overline{f(}(h, E, \bar{T}),\right. \\
z_{2} & =\bar{T}-\bar{D}(h, E), h \text { and } \bar{T} \text { satisfying Eq. (6) }\}
\end{aligned}
$$

Using Eq. (7) it is easy to see that the OSS problem corresponding to Eqs. (4-6) is to minimize $J_{S S}$ subject to

$$
J_{S S}=z_{1} ; z_{2}=0,\left(z_{1}, z_{2}\right) \epsilon \gamma(E), \quad E \geq 0
$$

The optimum solution of Eq. (8) is the same as the solution of the OSS problem for Eqs. (1-3); i.e., it is the classical optimum cruise. The optimum RSS solution of Eqs. (4-6) is obtained ${ }^{7,8,11}$ by minimizing $J_{R S S}$ subject to

$$
J_{R S S}=z_{1} ; z_{2}=0,\left(z_{i}, z_{2}\right) \epsilon \operatorname{co\gamma }(E), \quad E \geq 0
$$

where $\operatorname{co\gamma }(E)$ is the convex hull of $\gamma(E)$. If the optimal cost associated with Eq. (9), $J_{R S S^{*}}$, is less than the optimal cost associated with Eq. (8), $J_{S S^{*}}$, the OPC problem of Eqs: (4-6) is proper. ${ }^{7,8,11}$

\section{The F-4 Example}

For the F-4 aircraft it has been noted ${ }^{2}$ that $\gamma(E)$ is not convex. This means $J_{R S S}{ }^{*}<J_{S S}{ }^{*}$ is a possibility. To examine this possibility more closely, let $J_{S S}^{*}(E)$ and $J_{R S S}^{*}(E)$ denote, respectively, the minimum of $J_{S S}$ and $J_{R S S}$ in Eqs. (8) and (9) for fixed $E$. Figure 1 shows the results of numerical computations for the F-4 using the aerodynamic characteristics for airplane 1 in Bryson, Desai, and Hoffman. ${ }^{1}$ Military thrust (without afterburner) data are taken from Stephen and Chandler. ${ }^{12}$ The specific fuel rate is taken as $0.6(1+M) \mathrm{hr}^{-1}$ with dependency on Mach number $M$.

The point labeled 1 is the optimal classical cruise giving $J_{S S}^{*}$ $=7.505 \mathrm{lb} /$ mile. The point labeled 2 is the optimal relaxed cruise giving $J_{R S S}^{*}=7.379 \mathrm{lb} / \mathrm{mile}$, which is $1.7 \%$ less. If the same specific fuel rate is used with full afterburner thrust, this calculated improvement is larger. The relaxed cruise corresponds to infinitely rapid switching of $h$ and $V$ at constant $E$ between a zero-thrust, maximum-range glide condition $^{1}(h=25.0 \mathrm{kft}$ and $V=719 \mathrm{ft} / \mathrm{s})$ and essentially the maximum-thrust, minimum-fuel climb condition ${ }^{1}(h=21.1$ $\mathrm{kft}$ and $V=876 \mathrm{ft} / \mathrm{s}$ ). In practice this cannot be achieved, since it is impossible to interchange potential energy $(h)$ and kinetic energy $\left(V^{2} / 2 g\right)$ instantaneously as assumed by the energy-state approximation. A reasonable approximation to the optimum RSS cruise is a four-segment cycle where $E$ is allowed to vary slightly (say $E=33 \pm 2 \mathrm{kft}$ ) and the transitions between the zero-thrust condition and the maximum-thrust condition take place at a flight path angle of $\pm 45 \mathrm{deg}$. Approximate calculations show one cycle would consist of 1) a 61 sec zero-thrust glide from $h=26.5 \mathrm{kft}$ to $h=23.3 \mathrm{kft}, 2$ ) an 8 sec dive to $h=19.0 \mathrm{kft}, 3$ ) a $29 \mathrm{sec}$ maximum-thrust climb to $h=23.3 \mathrm{kft}$, and 4) a $6 \mathrm{sec}$ climb back to $h=26.5 \mathrm{kft}$. In view of the fairly small difference between $J_{R S S}^{*}$ and $J_{S S}^{*}$, it is not clear whether or not the improvements suggested for this four-segment trajectory would be maintained in a more accurate aircraft model, e.g., Eqs. (1-3). This conclusion agrees with the position taken by Zagalsky, et al. ${ }^{2}$

If a constraint on maximum $h$ is imposed, the potential improvement is much greater. For instance, a constraint $h \leq 10$ kft gives optimum points 3 and 4 in Fig. 1. The improvement between $J_{S S}^{*}=8.611 \mathrm{lb} / \mathrm{mile}$ and $J_{R S S}^{*}=7.979 \mathrm{lb} / \mathrm{mile}$ is then $7.3 \%$. Under the same assumptions as above $(E=13 \pm 2 \mathrm{kft}$ with transitions at $\gamma \pm 45 \mathrm{deg}$ ), the four-segment trajectory corresponding to point 4 would consist of 1) an 81 sec zerothrust glide from $h=10 \mathrm{kft}$ to $6.6 \mathrm{kft}, 2$ ) a $12 \mathrm{sec}$ dive to $h=0.85 \mathrm{kft}, 3$ ) a $17 \mathrm{sec}$ maximum-thrust climb to $4.6 \mathrm{kft}$, and 4) an $11 \mathrm{sec}$ climb back to $h=10 \mathrm{kft}$.

\section{An Idealized Aircraft Model}

Is the RSS improvement observed in the F-4 unusual? The discussion of Schultz and Zagalsky ${ }^{3}$ (Appendix A, Sec. 5) hints that it is not. The question will now be explored more fully by considering an idealized aircraft model in which the aerodynamic and engine data have the following form:

$$
\begin{gathered}
L=1 / 2 \rho S V^{2} C_{L_{\alpha}} \alpha ; D=1 / 2 \rho S V^{2}\left(C_{D_{0}}+\eta C_{L_{\alpha}} \alpha^{2}\right) \\
\rho=\rho_{0} e^{-h / h_{S}}, \sigma=\mathrm{const} ; T_{\max }=T_{0} e^{-h / h_{S}}
\end{gathered}
$$

The nomenclature for $C_{L_{\alpha}}, \mathrm{C}_{\mathrm{D}_{0}}, \eta, S$, and $\rho$ is standard; $h_{s}$ is the scale height of the exponential atmosphere; $\rho_{0}$ and $T_{0}$ are the density and thrust limit at $h=0 ; C_{L_{\alpha}}, C_{D_{0}}$, and $\eta$ are constant. While Eq. (10) is not claimed to be highly accurate it reflects in a simple analytic way the essential dependencies of $L, D$, and $T_{\max }$ in the subsonic case. By making the following definitions

$$
\begin{aligned}
& E=\ell \bar{E} ; h=\ell \bar{h} ; x=\ell \bar{x} ; T_{0}=W \bar{T}_{0} ; J=\sigma W(2 g \ell)^{-1 / 2} \bar{J} \\
& \ell=W\left(g \rho_{0} S\right)^{-1}(\eta)^{1 / 2}\left(C_{D_{0}} C_{L_{\alpha}}\right)^{-1 / 2} \\
& \beta=\ell\left(h_{s}\right)^{-1} ; \delta=\left(C_{D_{0}} \eta\right)^{1 / 2}\left(C_{L_{\alpha}}\right)^{-1 / 2}
\end{aligned}
$$

Eqs. (4) and (5) can be written:

$$
\begin{gathered}
\mathrm{d} \bar{E} / \mathrm{d} \bar{x}=\bar{T}-\delta\left[e^{-\beta \bar{h}}(\bar{E}-\bar{h})+e^{\beta \bar{h}}(\bar{E}-\bar{h})^{-1}\right] ; \bar{E}(0)=\bar{E}\left(\bar{x}_{1}\right) \\
\bar{J}=\frac{1}{\bar{x}_{I}} \int_{0}^{\bar{x}_{I}} \bar{T}(\bar{E}-\bar{h})^{-1 / 2} \mathrm{~d} \bar{x}
\end{gathered}
$$

The classical cruise for fixed $\bar{h}$ can be obtained from Eq. (12) and (13) by assuming $\mathrm{d} \bar{E} / \mathrm{d} \bar{x}$ is zero and minimizing $\bar{J}$ with respect to $\bar{E}$. Assuming that constraints on $T$ and $\alpha$ are not active, this yields

$$
\bar{J}_{S S}(\bar{h})=4(3)^{-3 / 4} \delta e^{-\beta \bar{h} / 2} ; \bar{T}=4(3)^{-1 / 2} \delta ; \bar{E}=\bar{h}+(3)^{1 / 2} e^{\beta \bar{h}}
$$

where $\bar{J}_{S S}(\bar{h})$ is the optimum cost at altitude $\bar{h}$. Thus, steadystate cruise performance is optimized by maximizing $\bar{h}$. In the model [Eq. (10)] there is a limit placed on $\bar{h}$ by the exponential decrease of $T_{\max }$. Thus, $\overline{h_{\max }}$ is that altitude at which the optimum thrust is the maximum possible; i.e., $\overline{h_{\max }}$ is determined by $4(3)^{-1 / 2} \delta=\bar{T}_{0} e^{-\beta \hbar}$.

It is not difficult to show that, for this problem, RSS concontrol cannot improve performance, i.e., $\bar{J}_{S S}^{*}=\bar{J}_{S S}\left(\bar{h}_{\max }\right)=$ $\bar{J}_{R S S}$. However, if the altitude is constrained to be less than $h_{\max }, \bar{h} \leq \bar{h}_{0}<\bar{h}_{\max }$, an improvement does result. In what follows, it is assumed that $\overline{h_{0}}=0$. With the exponential atmosphere this choice of constraint altitude does not limit the generality. Thus $\overline{h_{0}}$ becomes a zero reference altitude and $\rho_{0}$ and $T_{0}$ are the density and thrust limit at the constraint altitude. It is assumed further that constraints on minimum $h$ and maximum $\alpha$ are not active, which is usually the case. Thus, the effective constraints on Eqs. (12) and (13) are:

$$
\bar{h} \leq 0,0 \leq \bar{T} \leq \bar{T}_{0} e^{-\beta h^{-}}
$$

For the system [Eqs. $(12,13$, and 15)], it is clear from Eq. (14) that $\tilde{J}_{S S}^{*}=4(3)^{-3 / 4} \delta$. An expression for $J_{R S S}^{*}$ can be derived without great difficulty because the linearity of Eqs. (12) and (13) in $\bar{T}$ imply that the relaxed control "chatters", between $\bar{T}=0$ and $\bar{T}=\bar{T}_{0} e^{-\beta \bar{h}}$. This turns out to be a chattering between a zero-thrust glide at $\vec{h}=0$ and a maximum- 


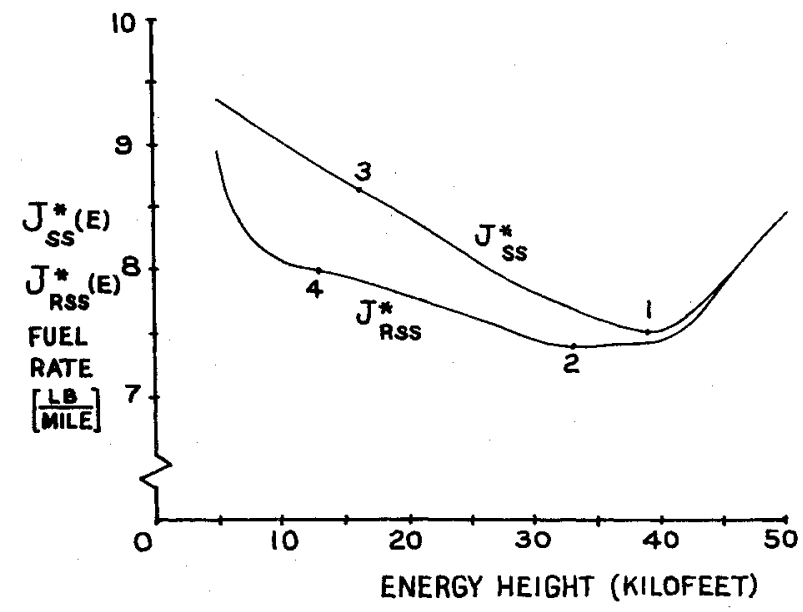

Fig. 1 Fuel consumption for $F-4$ in steady-state and relaxed steadystate cruise.

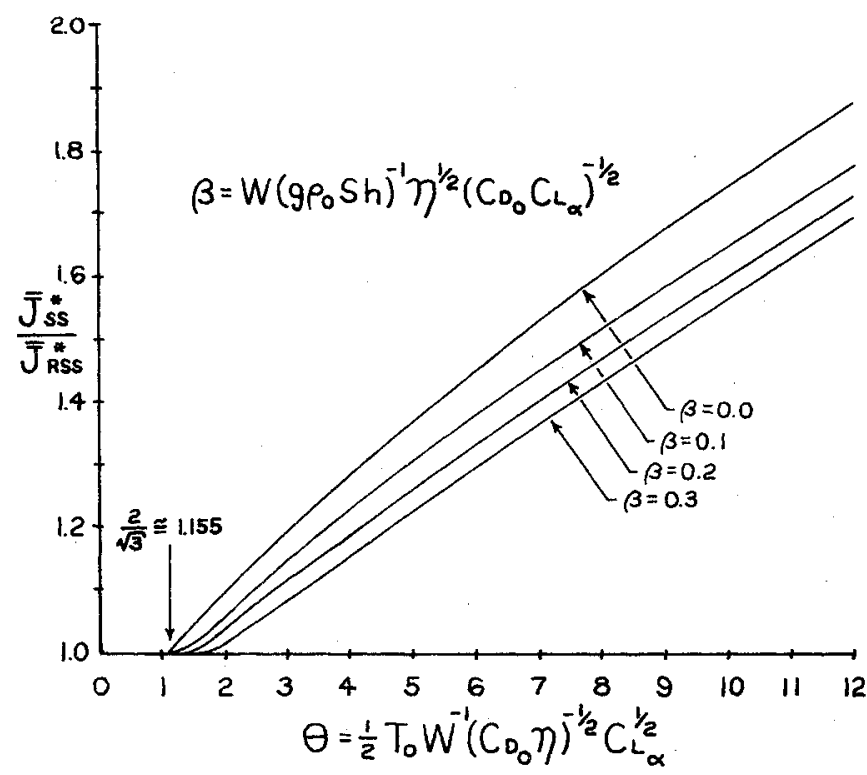

Fig. 2 Ratio of steady-state and relaxed steady-state cruise fuel rates.

thrust powered climb at $\bar{h}=\overline{h_{p}}$. For fixed $\bar{E}$ and $\overline{h_{p}}$ this leads to

$$
\begin{gathered}
\bar{J}_{R S S}=\bar{J}_{S S}^{*}(1 / 2)(3)^{3 / 4} \theta\left\{\psi ( \overline { E } ) \left[2 \theta-\left(\bar{E}-\overline{h_{p}}\right)\right.\right. \\
\left.\left.-\left(\bar{E}-\overline{h_{p}}\right)^{-1} e^{2 \beta \overline{h_{p}}}\right]+e^{\beta h_{p}}\right\}^{-1}\left(\bar{E}-\overline{h_{p}}\right)^{1 / 2}
\end{gathered}
$$

where

$$
\begin{gathered}
\psi(\bar{E})=\left(\bar{E}+\bar{E}^{-1}\right)^{-1} \text { for } \bar{E} \geq 1, \\
\psi(\bar{E})=1 / 2 \text { for } \bar{E}<1, \text { and } \theta=\bar{T}_{0}(2 \delta)^{-1}
\end{gathered}
$$

Minimizing $J_{R S S}$ with respect to $\tilde{E}$ and $\overline{h_{p}}$ determines $\bar{J}_{R S S}{ }^{*}$. This has been carried out numerically for different values of the nondimensional parameters $\theta$ and $\beta$ and the results are shown in Fig. 2.

For all $\theta>2(3)^{-1 / 2} \cong 1.155$ the RSS produces an improvement. For $\theta=2(3)^{-1 / 2}$ there is only enough thrust to produce the classical cruise, Eq. (14), at $\bar{h}=0$, and $\overline{J_{S S}^{*}}=\bar{J}_{R S S}^{*}$. For $\beta=0$, the optimum $\bar{E}=1$ for all $\theta$ and approximate formulas can be derived to give $\overline{h_{p}} \cong-(2 \theta-1) / 3$ and,

$$
\begin{aligned}
& \left(\bar{J}_{R S S}^{*}\right)^{-1} \bar{J}_{S S}^{*} \cong(2)^{5 / 2}(3)^{-9 / 4} \theta^{-1} \\
& \times(1+\theta)^{3 / 2}\left[1-9(1+\theta)^{-2 / 8}\right]
\end{aligned}
$$

The errors in this $\overline{h_{p}}$ and Eq. (17) are very small for $\theta \geq 5$; the worst errors are for $\theta<2$ and are about 0.2 for $\overline{h_{p}}$ and 0.001 for Eq. (17). For $\beta>0, h_{p}$ decreases slightly and $E$ depends on $\theta(\bar{E}<1.2$ for $\theta \geq 2$ and $0 \leq \beta \leq 0.3)$.
Figure 2 indicates the range of parameters where oscillatory aircraft motion is most likely to reduce fuel consumption. The most critical parameter is $\theta$, which can be interpreted as the ratio of maximum engine thrust to minimum drag, both evaluated at the constraint altitude. Thus low-drag, highthrust aircraft are favored. The parameter $\beta$ is affected most strongly by wing loading, and aircraft with low wing loading are favored.

\section{Conclusions}

It has been shown that the methodology of optimal periodic control is useful in analyzing the dynamics of aircraft cruise. Relaxed steady-state analysis suggests that oscillatory aircraft motion may reduce fuel consumption, particularly when altitude constraints are imposed. Aircraft with high thrust to drag ratios and low wing loading are favored.

Proving conclusively that substantial improvements can be attained requires additional analysis and computations with more elaborate aircraft models. This work is under way and will be reported in the future.

\section{References}

${ }^{1}$ Bryson, A.E., Jr., Desai, M.N., and Hoffman, W.C., "EnergyState Approximation in Performance Optimization of Supersonic Aircraft," Journal of Aircraft, Vol. 6, Nov.-Dec. 1969, pp. 481-488.

${ }^{2}$ Zagalsky, N.R., Irons, R.P., Jr., and Schultz, R.L., "Energy State Approximation and Minimum-Fuel Fixed-Range Trajectories," Journal of Aircraft, Vol. 8, June 1971, pp. 488-490.

${ }^{3}$ Schultz, R.L. and Zagalsky, N.R.," "Aircraft Performance Optimization," Journal of Aircraft, Vol. 9, Feb. 1972, pp. 108-114.

${ }^{4}$ Speyer, J.L., "On the Fuel Optimality of Cruise," Journal of Aircraft, Vol. 10, Dec. 1973, pp. 763-765.

${ }^{5}$ Schultz, R.L., "Fuel Optimality of Cruise," Journal of Aircraft, Vol. 11, Sept. 1974, pp. 586-587.

${ }^{6}$ Speyer, J.L., "A Test for Fuel Optimality of the Steady-State Cruise," to appear in the AIAA Journal.

${ }^{7}$ Bailey, J.E., "Periodic Operation of Chemical Reactors: A Review," Chemical Engineering Communications, Vol. 1, 1973, pp. 111-124.

${ }^{8}$ Guardabassi, G., Locatelli, A., and Rinaldi, S., "Status and Periodic Optimization of Dynamical Systems," Journal of Optimization Theory and Applications, Vol. 14, July 1974, pp. 1-20.

${ }^{9}$ Gilbert, E.G., "Vehicle Cruise: Improved Fuel Economy by Periodic Control," to appear in Automatica, 1976.

${ }^{10}$ Bittani, S., Fronza,G., and Guardabassi, G., "Periodic Control: A Frequency Domain Approach,"' IEEE Transactions on Automatic Control, Vol. AC-18, Feb. 1973, pp. 33-38.

${ }^{11}$ Bailey, J.E. and Horn, F.J.M.," Comparison Between Two Sufficient Conditions for Improvement of an Optimal Steady-State Process by Periodic Operation,' Journal of Optimzation Theory and Applications, Vol. 7, May 1971, pp. 378-389.

${ }^{12}$ Stephen, P.W. and Chandler, W.J., "Simultation Evaluation of Flight Command Functions," Hughes Aircraft Co., Los Angeles, Calif., Report AFFDL-TR-70-4, March 1970

\section{Monitoring Wake Vortex Strength Decay Near the Ground}

\author{
James N. Hallock* \\ DOT/Transportation Systems Center, \\ Cambridge, Mass.
}

\footnotetext{
A part of an extensive program ${ }^{1,2}$ to monitor the A behavior of wake vortices in the terminal environment, the strength or circulation of vortices is being determined for aircraft landing on runway $31 \mathrm{R}$ at the John F. Kennedy International Airport in New York. An array of monostatic

Presented as Paper 75-882 at the AIAA 8ch Fluid and Plasma Dynamics Conference, Hartford, Conn., June 16-18, 1975; submitted June 30, 1975; revision received March 31, 1976.

Index category: Jets, Wakes, and Viscid-Inviscid Flow Interactions.

${ }^{*}$ Engineer, Wake Vortex Program. Member AIAA.
} 